\title{
Effects of metal filament's alignment on tensile and electrical properties of conductive hybrid cover yarns
}

\author{
Ali Asghar ${ }^{1,2}$, Mohd Rozi Ahmad $^{1 *}$ and Mohamad Faizul Yahya ${ }^{1}$
}

${ }^{*}$ Correspondence: rozitex@salam.uitm.edu.my

${ }^{1}$ Faculty of Applied Sciences, Universiti Teknologi MARA,

Shah Alam, Selangor,

Malaysia

Full list of author information

is available at the end of the article

\begin{abstract}
Of late, a significant amount of research has been carried out using metals for conductive fabrics and composites to obtain the desired level of electromagnetic shielding along with physical and mechanical properties for its durability. However, incorporating these metallic filaments as core or as an integral part of core deteriorate the mechanical properties of textile yarns and ultimately the fabrics.' Moreover, the transparency of fabrics to high frequency/smaller wavelength waves further increases with the attempt to increase the metallic filament diameter. This study, therefore analyses the effects of metallic filament alignment in order to improve the mechanical properties of the resultant hybrid yarns, and devises an alternative method to increase the amount of conductive filament without increasing the diameter of the conducting wire in hybrid yarn structures. The results suggest that the tensile properties of the proposed hybrid cover yarns with conductive filament as covering component is superior as compared to the yarns having a conductive filament in the core, however, the electrical resistance increases with an increase in conductive filament length. The tenacity, elongation and initial modulus values were enhanced several times by incorporating the conductive filament as spiral covering. Moreover, with the proposed alignment method, the amount of copper in hybrid yarn can be increased up to some extent by changing the number of turns of covering component.
\end{abstract}

Keywords: Copper, Conductive yarns, Electrical resistance, Hybrid cover yarns, Metallic filament

\section{Introduction}

With the advent in technology, the reduction in the size of circuits has created smaller but more powerful sources for electromagnetic (EM) radiation emissions. These electronic/electrical devices have now surrounded our everyday environment in close proximity. The radiated emissions are not just responsible for electromagnetic interference (EMI) in between the devices but also have negative influences on human health (Jagatheesan et al. 2014). The electromagnetic radiation is already categorized as the fourth most serious source of public pollution following the noise, air and water pollution (Ozen et al. 2012). This pollution is mainly attributed to the ever-increasing use of wireless communications all around the globe. With the further advancement of information

(c) 2016 Asghar et al. This article is distributed under the terms of the Creative Commons Attribution 4.0 International License (http://creativecommons.org/licenses/by/4.0/), which permits unrestricted use, distribution, and reproduction in any medium, provided you give appropriate credit to the original author(s) and the source, provide a link to the Creative Commons license, and indicate if changes were made. 
technology, and in order to cope up with the needs, the useable range of EM wave's frequencies will shift to much higher bandwidths and thus will be more problematic as much higher levels of this pollutant will spread in the atmosphere. In order to cope up with the higher frequencies, significant amount of research has been carried out using metals to conductive fabrics and composites to obtain the desired levels of shielding as well as physical and mechanical properties for its durability (Jagatheesan et al. 2014).

Conductive yarns, as per their name sounds, are capable of conducting electricity through them. Usually the textile materials (natural and synthetic), at standard atmospheric conditions, are very good electrical insulators with the exception of only few intrinsically conductive polymers such as polyacetylene, polyaniline, polypyrrole etc., which are capable of conducting charges (Maity et al. 2013). Non-conducting textile materials can be converted to conductive materials by either giving them surface treatments like conductive paints, ionic/electroless plating, vacuum metallization or by incorporating conductive fillers inside in a particular order (Cheng 2006). According to Duran and Kado lu (2014), certain properties of conductive textiles that includes: weight, flexibility, pliability, air permeability, porosity, comfort in wearing, less corrosiveness and lower cost of production, have made its use imperative in applications where electromagnetic shielding and static dissipation is required.

There are certain limitations to every method for the production of conductive fabrics; the limiting factors for intrinsically conductive polymers are poor long-term stability, washing fastness and rigid characteristics (Šaravanja et al. 2015). Similarly, coating of carbon fibres onto a material for conductivity is reported to have very high cost of production, whereas, the use of Carbon Nano tubes is restricted for its uniformity and dispersibility, specially for the Multi-walled Carbon Nano tubes (Jagatheesan et al. 2014). Although it has its own demerits, but producing fabric by incorporating conductive fillers (hybrid yarns) inside is a very popular and common way of producing EM shielding fabrics.

These conductive fillers or hybrid yarns can be formed by using continues filaments like stainless steel or copper wires placed alongside (plied) with non-conducting textile materials, placed as core material with covering of textile materials or in the form of discontinuous cut staple fibres (limiting factor), blended together with some non-conductive natural or synthetic textile materials. Rajendrakumar and Thilagavathi (2012), plied polyester filament with $0.04 \mathrm{~mm}$ copper wire on a ring doubling machine to investigate the effects of fabric weave and pick density on electromagnetic shielding effectiveness (EMSE). They concluded that the plain weave posed better EMSE as more number of interlacements reduces the contact resistance, they further concluded that the pick density is directly related to increased EMSE.

Duran and Kado lu (2014), inserted silver coated Polyamide filaments in core via a special arrangement after the front roll delivery system in Ring spinning. They used this hybrid yarn structure in the weft with varying weft densities to analyse its impacts on EMSE, moreover, they investigated different proportions of $\mathrm{Ag} / \mathrm{PA}$ and concluded that the higher the \% age of conductive material in blend the higher will be the EMSE. Several other researchers used somewhat similar techniques to incorporate the conductive filament in core, like, Yu et al. (2014), inserted stainless steel wire of $50 \mu \mathrm{m}$ in core by using hollow spinning machine, the covering components were Antibacterial Nylon and 
Bamboo Charcoal Polyester filament. Siro core spinning system with double roving as outer layers and stainless steel core was examined for the effects of orientation of fibres within the structure by Ortlek et al. (2012). Recently, Yu et al. (2015), incorporated stainless steel wire in core using hollow spindle spinning in order to investigate the functional and EMSE of elastic warp knitted fabrics. They concluded that multi-layered fabrics with laminating angles $0^{\circ} / 90^{\circ}$ produced better results as compared to $0^{\circ} / 0^{\circ}$ layered fabrics due to the formation of metal grids in the arrangement.

Liu and Rong (2015), investigated the effects of metal fibre contents by incorporating a blended yarn having $25 \%$ stainless steel fibres into fabrics with varying warp and weft densities. They concluded that the greater the metal fibre content of a fabric more would be the conductivity and better EMSE.

In general, metal filament/wires have rigid characteristics when compared to textile yarns and filaments. They are resilient and have less efficiency to return to their normal shapes after experiencing mechanical deformations. Moreover, when placed inside the yarn as core component (in case of core-sheath or plied yarns), the mechanical/tensile properties of these metallic filaments drape over the properties of the textile component (Vasile et al. 2011). Since, the core component's mechanical/tensile properties in any hybrid yarn structure dominates the yarn's mechanical/tensile properties ( $\mathrm{Pl}$ et al. 2015), therefore incorporating the metallic filaments as core deteriorate the mechanical/tensile properties of textile yarns and ultimately the fabrics'.

Furthermore, the rigidity of these metallic wires, when inserted as core component, causes openness in fabrics as they are not properly interlaced, hence making the fabric transparent for the smaller wavelength waves (Cheng 2006). The transparency of fabrics to high frequency/smaller wavelength waves further increases with the attempt to increase the metallic wire's diameter, in order to increase the amount of metal inside the fabrics for greater EM shielding effectiveness (Perumalraj and Dasaradan, 2010).

These declining mechanical/tensile properties due to the insertion of metallic filaments in the hybrid yarn's core have been neglected by the researchers so far. Their studies were mainly concentrated around improving the electrical conductance/EM shielding. Moreover, the increase in diameter of the metallic core to increase the EMSE further aggravates the problem. This study therefore analyses the effects of metallic filament's alignment in order to improve the mechanical properties of the resultant hybrid yarns and devises an alternative method to increase the amount of conductive filament without increasing the diameter of the conductive wire in hybrid yarn structures.

\section{Methods}

Materials

Two different genres of copper with respect to their diameters that includes 0.1 and $0.2 \mathrm{~mm}$ with Tex counts of 75 and 320.80 Tex respectively, were used as conductive components for the study. The copper wires were obtained from the open market manufactured by FE Magnet Wire Company, Malaysia. The electrical resistance was recorded as $2300 \Omega / \mathrm{KM}$ for $0.1 \mathrm{~mm}$ diameter and $687 \Omega / \mathrm{KM}$ for $0.2 \mathrm{~mm}$ diameter. For the counter non-conductive textile part, 31.60 Tex Polyester yarn and 78.52 Tex Acrylic/Wool (AW) yarn (blending ratio of 70/30) were used. The selection of non-conductive textile component such as Polyester and Acrylic/Wool was carried out just to highlight the impacts 
of alignment of components regardless of their fibre type. Winding of $0.2 \mathrm{~mm}$ copper on to a core require the core to be thick or bulky enough for the wire to wound properly. Coarse count AW yarn was selected as it provides the required bulkiness.

\section{Preparation of yarn samples}

The two component hybrid cover yarns were produced using a hollow spindle-spinning machine YCHN-303. The two components include a core component and a cover component (Figs. 1, 2). The core component was drawn through the machine's drafting arrangement (without being drafted) from within the hollow spindle to the output roller, whereas, the covering component was wound on hollow spindle's bobbin. Different yarn samples were produced by varying the genre and alignment of conductive and nonconductive components and are represented in Tables 1 and 2. The machine parameters were carefully monitored and controlled as it has considerable influence on the output product. These parameters include the hollow spindle speed, tension of core component and machine throughput for controlling and varying the number of turns of covering.

\section{Tensile testing of yarn}

For the tensile properties of conductive hybrid cover yarns, Testometric Tensile tester was utilized following the guidelines of ASTM D2256. According to the standard, the sample breaking time of $20 \pm 3 \mathrm{~s}$ was achieved at the extension rate of $100 \mathrm{~mm} / \mathrm{min}$ on the CRE type machine. The load cell used had the maximum loading capacity of $500 \mathrm{~N}$, which is recommended for yarn testing according to the standard. The recommended
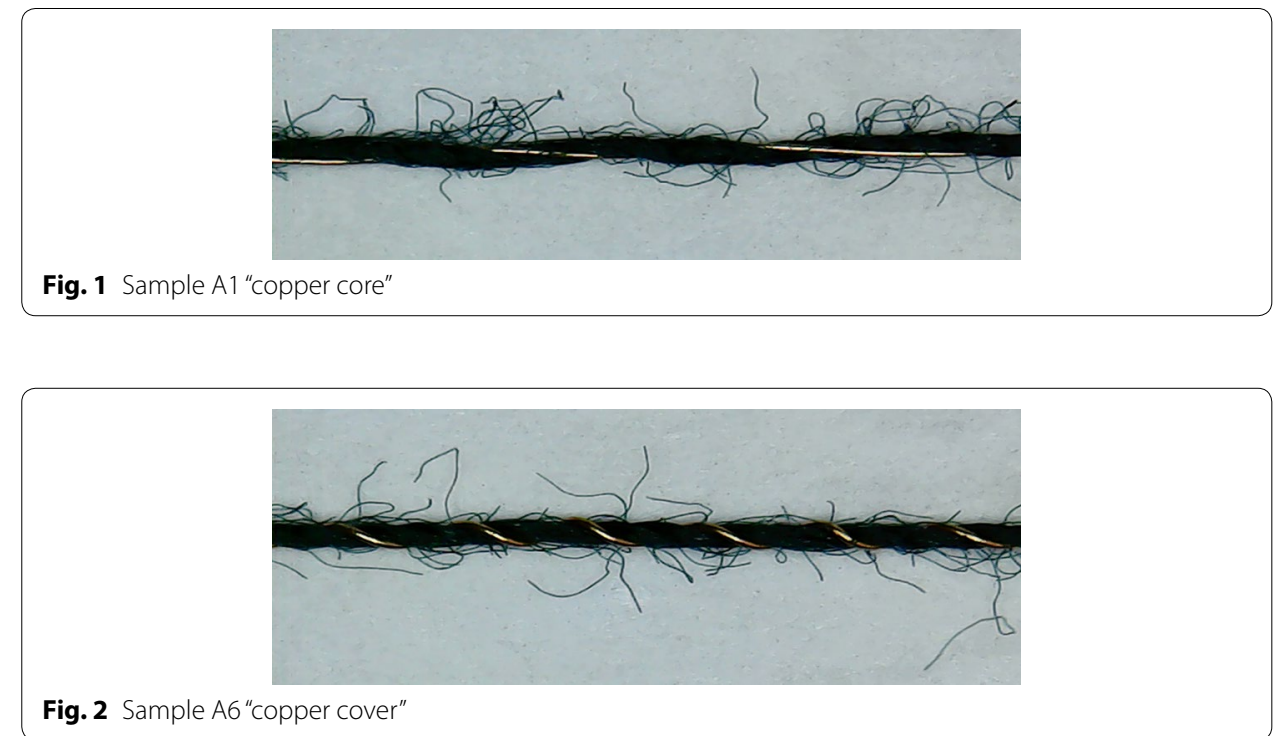

Table 1 Component definition

\begin{tabular}{llc}
\hline Yarn sample ID & Non-conductive textile component & Conductive component \\
\hline A & 31.61 Tex polyester yarn 40/2 cotton count & $\begin{array}{c}0.1 \mathrm{~mm} \text { dia, 75 Tex cop- } \\
\text { per wire }\end{array}$ \\
B & 78.52 Tex AW yarn (70/30) 28/2 worsted count & $\begin{array}{c}0.2 \mathrm{~mm} \mathrm{dia} \text {, 320.80 Tex } \\
\text { copper wire }\end{array}$ \\
\hline
\end{tabular}


Table 2 Sample classification

\begin{tabular}{lllllll}
\hline $\begin{array}{l}\text { Yarn sample } \\
\text { number }\end{array}$ & $\begin{array}{l}\text { Core } \\
\text { component }\end{array}$ & $\begin{array}{l}\text { Covering } \\
\text { component }\end{array}$ & $\begin{array}{l}\text { Turns per } \\
\text { meter of } \\
\text { covering } \\
\text { component } \\
\text { (TPM) }\end{array}$ & $\begin{array}{l}\text { Resultant } \\
\text { count } \\
\text { (Tex) }\end{array}$ & $\begin{array}{l}\text { Amount of } \\
\text { core (\% age) }\end{array}$ & $\begin{array}{l}\text { Amount of } \\
\text { covering } \\
\text { (\% age) }\end{array}$ \\
\hline A1 & Copper wire & Polyester yarn & 342 & 89.40 & 62.34 & 37.66 \\
A2 & Polyester yarn & Copper wire & 429 & 109.45 & 27.85 & 72.15 \\
A3 & Polyester yarn & Copperwire & 504 & 111.82 & 27.64 & 72.36 \\
A4 & Polyester yarn & Copperwire & 630 & 112.15 & 26.85 & 73.15 \\
A5 & Polyester yarn & Copper wire & 771 & 115.50 & 27.15 & 72.85 \\
A6 & Polyester yarn & Copper wire & 941 & 116.72 & 26.38 & 73.62 \\
B1 & Copper wire & AW yarn & 512 & 354.56 & 73.10 & 26.90 \\
B2 & AW yarn & Copperwire & 354 & 415.32 & 18.27 & 81.73 \\
B3 & AW yarn & Copperwire & 433 & 420.32 & 18.39 & 81.61 \\
\hline
\end{tabular}

type of yarn clamping jaws with the gauge setting of $250 \mathrm{~mm}$ was used. The yarns were tested and compared to their basic tensile properties, which includes tensile strength, elongation and initial modulus.

\section{Electrical resistance testing}

AATCC Test Method 84-2011 was used as the standard procedure for testing the electrical resistance of yarns. KEITHLEY 197 Autoranging Microvolt DMM meter was used for analysing the electrical resistance of $100 \mathrm{~mm}$ yarn samples at standard test atmospheric conditions. Since copper wires have very less resistance to the flow of electricity, measurements at $200 \Omega$ range and $450 \mathrm{~V}$ were conducted using a 4-Terminal configuration (Fig. 3) in order to minimize errors due to the voltage drop across the test leads. The Kelvin test leads into the input HI/LOW and OHMS SENSE HI/LOW were used. The test leads were shortcircuited at Relative (REL) mode to reveal $000.000 \mathrm{ohms}$ resistance (Fig. 4). The resistance meter was calibrated as per the procedures laid down by the operations manual.

\section{Results and discussions}

\section{Effects of metal filament's alignment on tensile properties}

\section{Tenacity}

It is evident from Fig. 5, that the non-conductive textile components have much higher tenacity values as compared to their counter conductive part. Polyester yarn with the

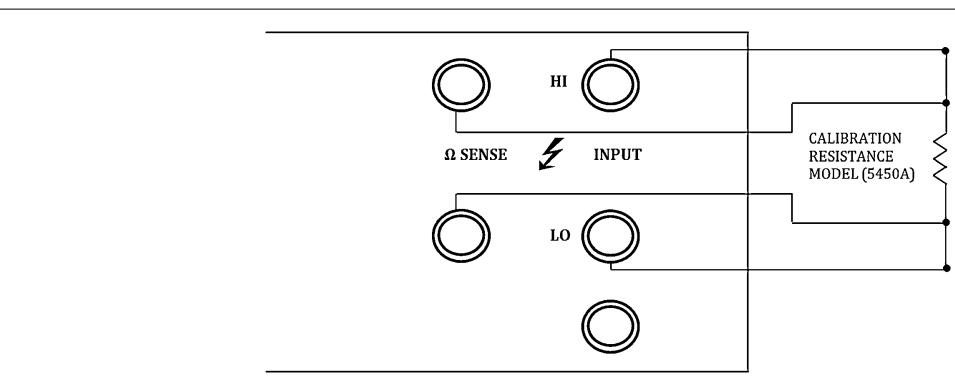

Fig. 3 4-Terminal configuration 


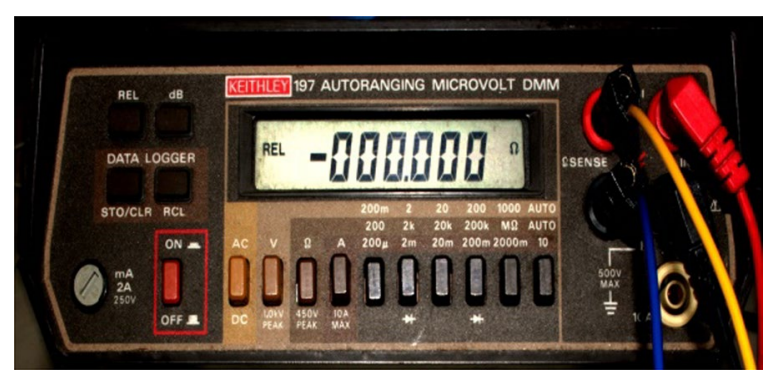

Fig. 4 Calibrated multimeter

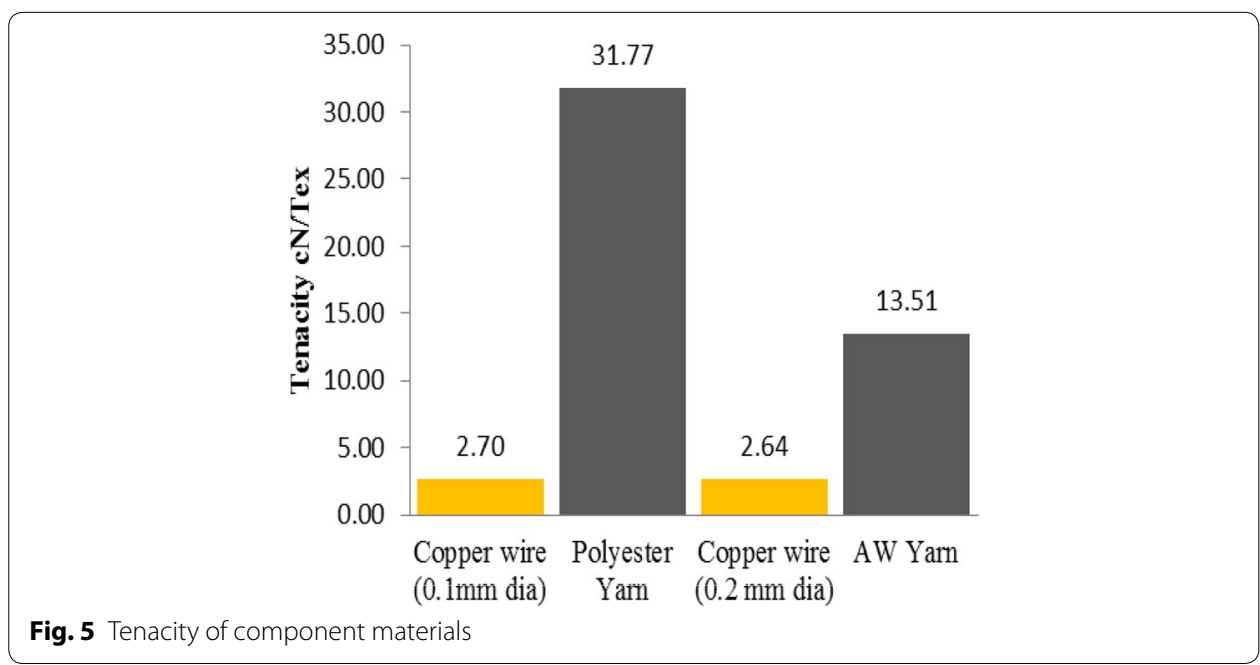

tenacity of $31.77 \mathrm{cN} /$ tex was pooled with $2.70 \mathrm{cN} /$ tex copper wire in two different alignments (Figs. 1, 2). The hybrid yarn sample A1, which shows the tenacity of $2.69 \mathrm{cN} /$ tex (Fig. 5), had copper wire as the core component. The spiral polyester yarn on the cover of A1, did not contribute at all to the tenacity of the hybrid yarn structure, in fact, it behaved like a spring that allowed the core to be stretched when axial load was applied. Hence, the sample A1 reflects the tenacity equivalent to the reference copper wire's tenacity.

Sample A2 and A3 have the opposite structural contents as of sample A1, that is, polyester core and copper (Fig. 6) covering with varying twists. The tenacity values for the hybrid yarn samples A2 and A3 are 11.01 and $10.33 \mathrm{cN} /$ tex respectively, which is around four times higher than the tenacity of sample A1.

A somewhat similar pattern was observed for AW and $0.2 \mathrm{~mm}$ copper hybrid yarns. The sample B1, that had copper as core, showed the tenacity value of $2.92 \mathrm{cN} / \mathrm{tex}$. The copper wire used for preparing B1 has the tenacity of $2.64 \mathrm{cN} /$ tex, which is transferred to the hybrid yarn sample. Again, the AW covering had no or very little contribution towards the tenacity of this hybrid yarn structure. Whereas, the samples B2 and B3, which had the AW core and copper covering, showed higher tenacity values than that of sample B1.

However, the samples A2, A3 and B2, B3 should have displayed the tenacity values almost equivalent to the tenacity of reference core yarns. The reason for reduced tenacity 


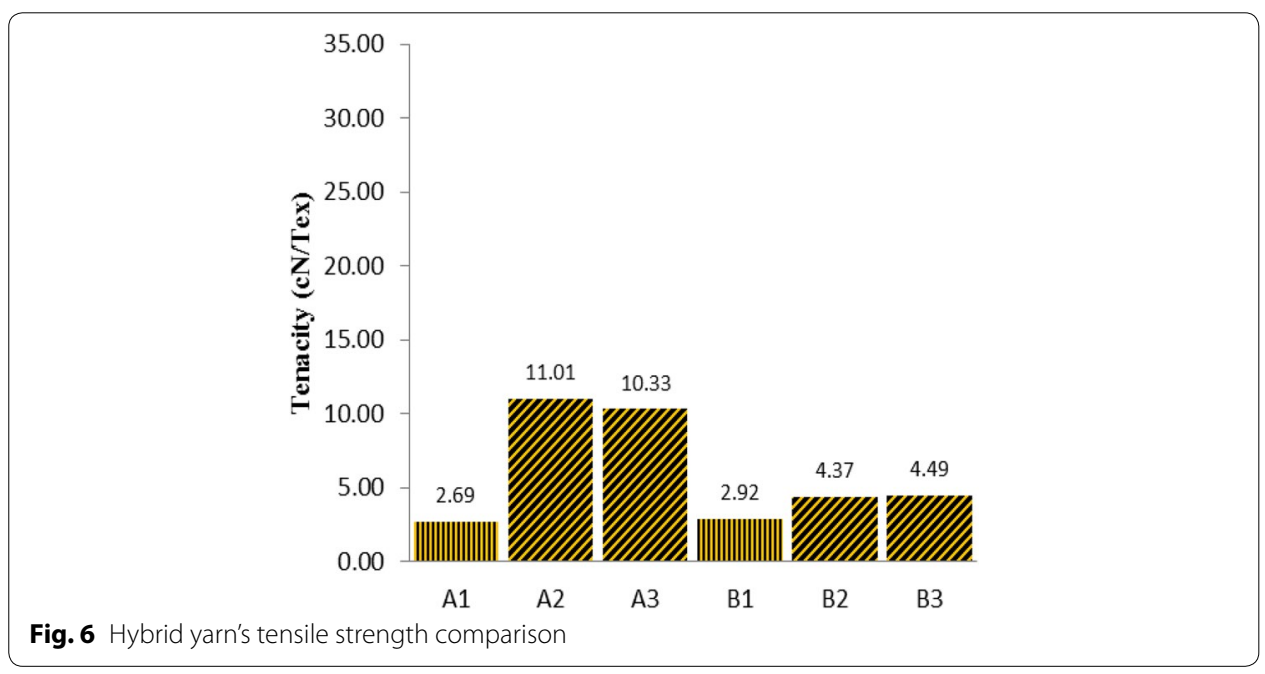

could be the resistance provided by the multiple coils of stiff copper wires. Generally, the more the number of coils the greater should be the tensile strength of the resultant hybrid yarn, since these coils get further tighten up and exert lateral pressure as the axial forces are applied. Therefore, yarn sample A6 with 941 coils per meter has better tenacity values than the two preceding samples as shown in Fig. 7. The R-squared value of 0.9483 verifies this second-degree polynomial regression.

\section{Elongation}

The elongation values of the component materials are displayed in Fig. 8. Both the conductive and non-conductive materials showed somewhat higher elongation values when measured separately. Whereas, when they are combined as hybrid yarns, the samples having copper as a core component (A1 and B1) showed very less elongation (Fig. 9), while the samples having polyester and AW yarn as core components (A2, A3, B2, B3) had very high elongation values (around 4-6 times higher).

During hybrid yarn preparation in hollow spinning, the core component had to be kept in high tension for winding the cover properly around the core. To achieve the required tension in case of copper core samples (A1 and B1), a draft of 1.3 between

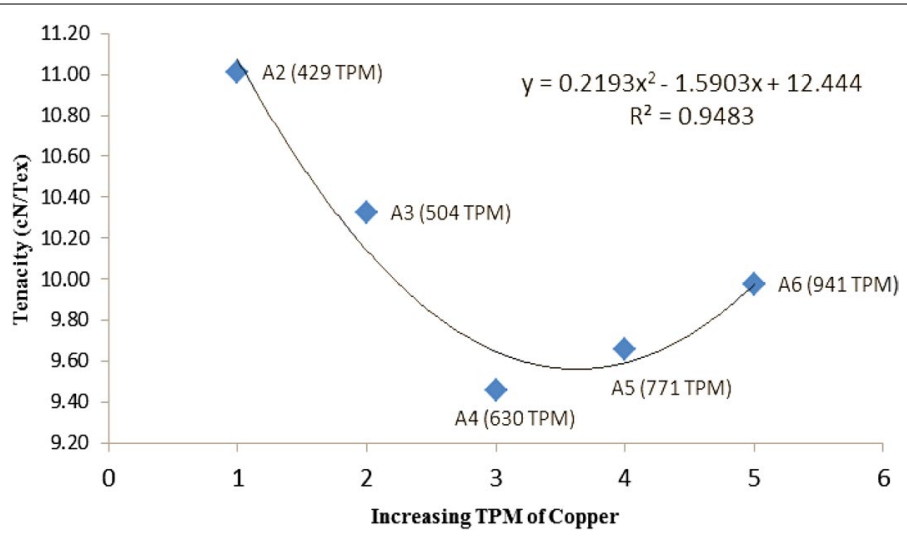

Fig. 7 Increasing TPM of copper and tenacity 

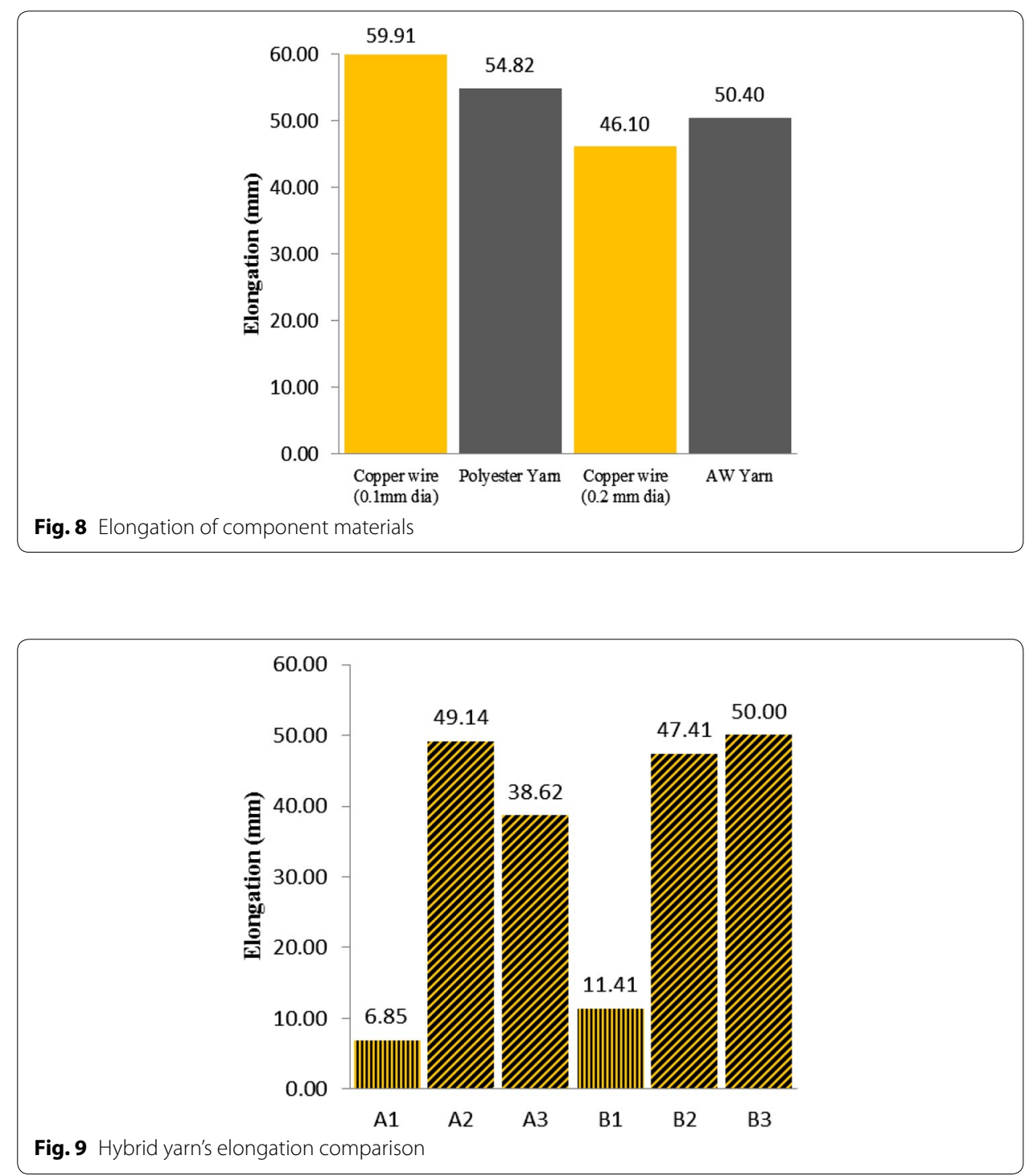

the front drafting roller and the take-up roller had to be maintained. Since copper has very less elasticity due to its high modulus, this draft during spinning made it permanently elongated, hence the elongation values in the hybrid yarn sample A1 and B1 were reduced. This is also reflected in the resultant count of the sample A1 and B1 in Table 2. The resultant count of 75 Tex copper wire and 31.60 Tex polyester turned out to be 89.40 Tex only (sample A1). Similarly, 320.80 Tex copper with 78.52 Tex AW yarn blend in hybrid yarn B1 was only 354.56 Tex.

\section{Initial modulus}

The initial modulus values of the component materials are displayed in Fig. 10, where copper showed very high modulus values as compared to the non-conductive textile components. When converted to hybrid yarn structure while keeping copper as a core component (A1 and B1), the initial modulus values of the resultant hybrid yarn remained high, hence making the yarn stiffer and less elastic. 


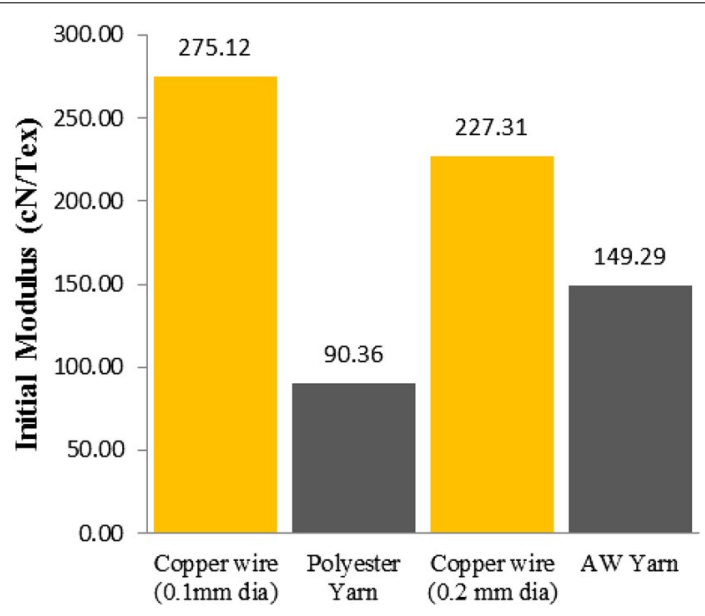

Fig. 10 Initial modulus of component materials

Samples A2, A3 and B2, B3 which had copper as covering material and non-conductive textile core, performed otherwise, i.e., showing less modulus values (Fig. 11). Copper as a spiral covering, did not contribute to the modulus values of these hybrid yarns as it can extend and relax itself like that of a helical spring when an axial load was applied. This elastic helical spring made from the coils of copper, even improves the modulus values of the hybrid yarn structure.

Although there was no significant relation found between turns per meter of copper and initial modulus in this research, however, the number of coils of spring does have a very significant relation to its elasticity, which further needs to be assessed.

\section{Effects of metal filament's alignment on amount of copper}

Figure 12 plotted for sample A2 to A6 represents the relation between turns per meter and copper weight in grams per $1000 \mathrm{~m}$. The R-squared value of 0.9825 for the regression line plotted between the data points clearly shows an increasing linear relation between TPM and increase in copper weight.

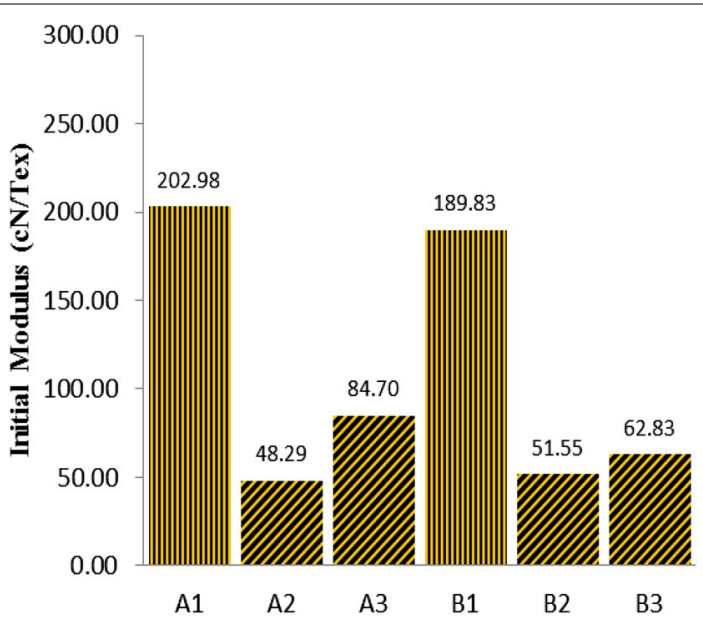

Fig. 11 Hybrid yarn's initial modulus comparison 


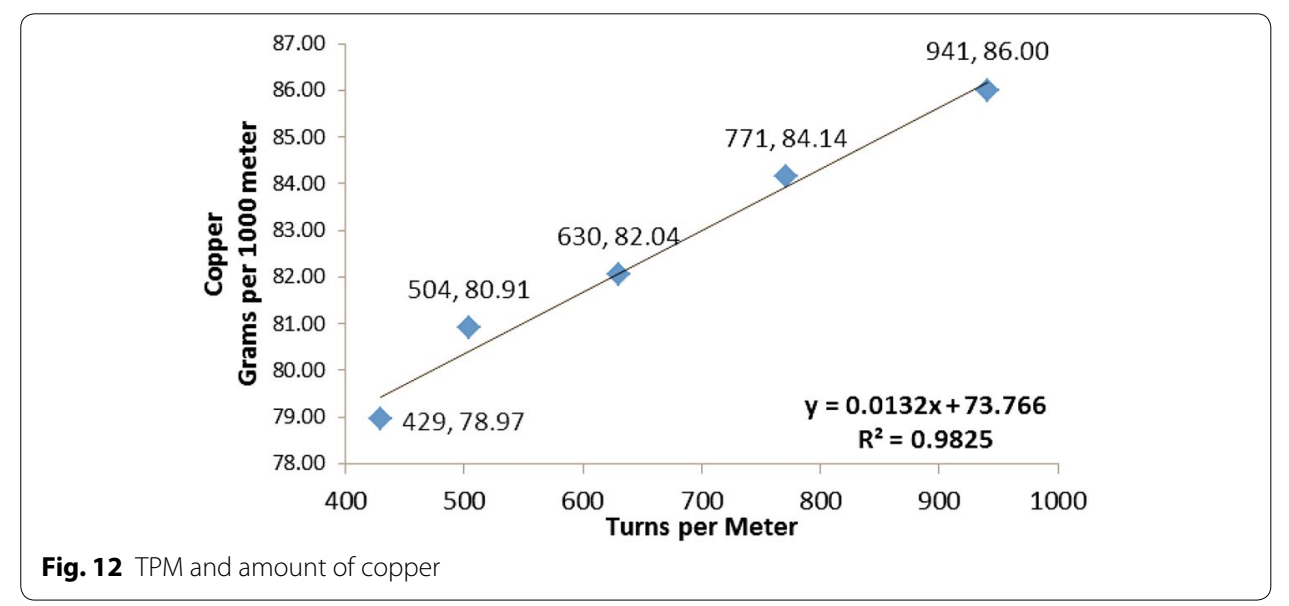

The values on Y-axis are in weight per unit length, i.e., grams per $1000 \mathrm{~m}$ or simply Tex. It can be seen from the figure that a 75 Tex copper can be converted to 86 Tex wire by increasing the number of coils to 941 turns per meter. The resultant Tex count of the hybrid yarn increases as shown in Table 2 (A2-A6), but since the diameter of the resultant yarn remains the same, hence various amounts of copper can be adjusted by changing the number of coils in the hybrid yarn structure for improving the electromagnetic shielding efficiency of the resultant fabrics.

\section{Effects of metal filament's alignment on electrical resistance}

Figure 13 represents the relation between the turns per meter and the electrical resistance in ohms per $100 \mathrm{~mm}$ of yarn samples at $25{ }^{\circ} \mathrm{C}$ and $65 \% \mathrm{RH}$ for samples $\mathrm{A} 2$ to A6. Although the data points are somewhat scattered along the regression line and the coefficient of determination of 0.8032 also verifies it, still there is an increasing linear relationship amongst the variables. Whereas, the increase in electrical resistance is not remarkable, i.e., only $6.4 \mathrm{e}^{-3} \Omega / 100 \mathrm{~mm}$ increases by increasing the TPM from 429 to 941. The increase in number of coils of copper in the sample A2 to A6 causes increase in length of the copper wire. This can also be verified from the resultant count increase

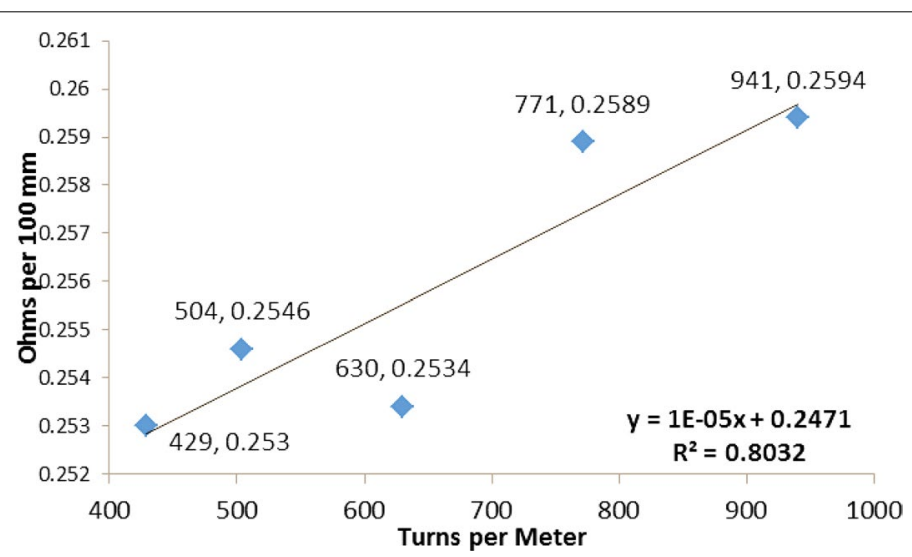

Fig. 13 TPM and electrical resistance of yarn 
Table 3 Electrical properties of hybrid cover yarns

\begin{tabular}{llc}
\hline Sample number & $\begin{array}{l}\text { Electrical resistance } \\
\text { (ohms per 100 mm) }\end{array}$ & $\begin{array}{l}\text { Amount of copper } \\
\text { (grams/1000 m) }\end{array}$ \\
\hline Copper wire (0.1 mm dia) & 0.2300 & 75.00 \\
A1 & 0.2468 & 55.73 \\
A2 & 0.2530 & 78.97 \\
A3 & 0.2546 & 80.91 \\
Copper wire (0.2 mm dia) & 0.0658 & 320.80 \\
B1 & 0.0687 & 259.20 \\
B2 & 0.0701 & 339.44 \\
B3 & 0.0784 & 343.10 \\
\hline
\end{tabular}

as all the other variables besides the TPM were kept constant. The electrical resistance increases with the increase in length of wire and decreases with the diameter. It can be observed from the Table 3 that the reference copper wire of $0.2 \mathrm{~mm}$ showed only 0.0658 $\Omega / 100 \mathrm{~mm}$ resistance as compared to $0.23 \Omega / 100 \mathrm{~mm}$ resistance of $0.1 \mathrm{~mm}$ copper wire.

Sample A1 (copper core) showed a resistance value of $0.2468 \Omega / 100 \mathrm{~mm}$ which is higher than the reference copper resistance of $0.23 \Omega / 100 \mathrm{~mm}$. Similarly, the sample B1 had $0.0687 \Omega / 100 \mathrm{~mm}$ resistance while $0.2 \mathrm{~mm}$ diameter copper wire had 0.0658 $\Omega / 100 \mathrm{~mm}$ resistance. This increase of resistance of Sample A1 and B1 is related to the permanent elongation of the copper wire caused by the draft in the delivery zone of the hollow spindle-spinning machine that eventually increased its resistance.

\section{Conclusions}

The tensile properties of the hybrid cover yarns with conductive filament as covering component is superior as compared to the yarns having a conductive filament in core. The tenacity, elongation and initial modulus values were enhanced several times by incorporating the conductive filament as spiral covering.

Moreover, the amount of copper in hybrid yarn can be altered up to some range by changing the number of turns of covering component. Hence, the disadvantage of incorporating larger diameter wires can be overcome up to certain extent.

The increased coils or TPM of copper has a negative influence as far as electrical resistance is concerned. However, the increase in electrical resistance was not remarkable.

The proposed conductive yarn when converted to fabrics will improve some of the mechanical properties like flexibility, pliability, strength and durability of the conductive fabrics; moreover, the fabric can be woven with higher cover factor to restrict the smaller wavelength waves since the diameter of conductive wires inside the fabrics need not to be enhanced.

\footnotetext{
Author details

${ }^{1}$ Faculty of Applied Sciences, Universiti Teknologi MARA, Shah Alam, Selangor, Malaysia. ${ }^{2}$ Baluchistan University of IT, Engineering and Management Sciences, Quetta, Pakistan.
}

Received: 12 October 2015 Accepted: 31 December 2015

Published online: 28 January 2016 


\section{References}

Cheng, K. B. (2006). Electromagnetic shielding effectiveness of the twill copper woven fabrics. Journal of Reinforced Plastics and Composites, 25(7), 699-709. doi:10.1177/0731684406060578.

Duran, D., \& Kado lu, H. (2014). Electromagnetic shielding characterization of conductive woven fabrics produced with silver-containing yarns. Textile Research Journal,. doi:10.1177/0040517512468811.

Jagatheesan, K., Ramasamy, A., Das, A., \& Basu, A. (2014). Electromagnetic shielding behaviour of conductive filler composites and conductive fabrics - a review. Indian Journal of Fibre and Textile Research, 39(September), 329-342.

Liu, Z., \& Rong, X. (2015). Influence of metal fibre content of blended electromagnetic shielding fabric on shielding effectiveness considering fabric weave. Fibers and Textiles in Eastern Europe, 4(112), 83-87. doi:10.5604/12303666.1155635.

Maity, S., Singha, K., Debnath, P., \& Singha, M. (2013). Textiles in electromagnetic radiation protection. Journal of Safety Engineering, 2(2), 11-19. doi:10.5923/j.safety.20130202.01.

Ortlek, H. G., Alpyildiz, T., \& Kilic, G. (2012). Determination of electromagnetic shielding performance of hybrid yarn knitted fabrics with anechoic chamber method. Textile Research Journal, 83(1), 90-99. doi:10.1177/0040517512456758.

Ozen, M. S., Sancak, E., Beyit, A., Usta, I., \& Akalin, M. (2012). Investigation of electromagnetic shielding properties of needle-punched nonwoven fabrics with stainless steel and polyester fiber. Textile Research Journal, 83(8), 849-858. doi:10.1177/0040517512461683.

Perumalraj, R., \& Dasaradan, B. S. (2010). Electromagnetic shielding effectiveness of doubled copper-cotton yarn woven materials. Fibers and Textiles in Eastern Europe, 18(3), 74-80.

PI, Öi., Mukavemet, I. KI. N., \& Kler, Öl. (2015). Tensile properties of some technical core spun yarns developed for protective textiles. Tekstil ve Konfeksiyon (Journal of Textile and Apparel), 25(2), 104-110.

Rajendrakumar, K., \& Thilagavathi, G. (2012). Electromagnetic shielding effectiveness of copper/PET composite yarn fabrics. Indian Journal of Fibre and Textile Research, 37(June), 133-137.

Šaravanja, B., Malarić, K., \& Pušić, T. (2015). Impact of dry cleaning on the electromagnetic shield characteristics of interlining fabric. Fibres and Textiles in Eastern Europe, 1(109), 104-108.

Vasile, S., Githaiga, J., \& Ciesielska-Wróbel, I. L. (2011). Comparative analysis of the mechanical properties of hybrid yarns with superelastic shape memory alloys (SMA) wires embedded. Fibres and Textiles in Eastern Europe, 89(6), 41-46.

Yu, Z., He, H., Lu, Y., Zhang, J., Lou, C., Chen, A., \& Lin, J. (2015). Functional properties and electromagnetic shielding behaviour of elastic warp-knitted fabrics. Fibers and Textiles in Eastern Europe, 5(113), 78-83. doi:10.5604/12303666.1161761.

Yu, Z.C., Lu, Y.H., He, H.L., Zhang, J.F., Lou, C.W., Chen, A.P., \& Lin, J.H. (2014). Antibacterial properties and electrical characteristics of multifunctional metal composite fabrics. Journal of Industrial Textiles, (100). http://doi. org/10.1177/1528083714542824

\section{Submit your manuscript to a SpringerOpen ${ }^{\circ}$ journal and benefit from:}

- Convenient online submission

- Rigorous peer review

- Immediate publication on acceptance

- Open access: articles freely available online

- High visibility within the field

- Retaining the copyright to your article

Submit your next manuscript at $\boldsymbol{s p r i n g e r o p e n . c o m ~}$ 